\title{
Surveillance for and issues relating to noncommunicable diseases post-Haiyan in Region 8
}

\author{
Rammell Eric Martinez, ${ }^{a}$ Ronaldo Quintana, ${ }^{a} J o h n$ Juliard Go, ${ }^{a}$ Mae Analyne Marquez, ${ }^{b}$ Jae Kyoun Kim, ${ }^{a}$ \\ Ma Sol Villones, ${ }^{b}$ and Miguel Antonio Salazar ${ }^{a}$ \\ Correspondence to Rammell Eric Martinez (email: martinezra@wpro.who.int or rammell.martinez@gmail.com).
}

D isasters complicate the management of noncommunicable diseases (NCDs) by disrupting access to and delivery of health care, including medicines. Following Typhoon Haiyan in the Philippines in November 2013, much of the health infrastructure was destroyed and health service delivery was severely affected in Region 8. This left many people with NCDs vulnerable as medicines were destroyed or washed away, food was scarce, and access to medicines and drugs and quality health care was difficult.

There is limited information about managing NCDs after disasters. Rapid health assessment tools do not tend to include questions about NCDs or the factors needed to respond to them post-disaster. ${ }^{1}$ Most available guidelines for the health sector response postdisaster focus primarily on safe drinking-water, food, sanitation and hygiene, acute medical conditions, acute malnutrition, communicable diseases and injury; guidelines on chronic conditions are limited. ${ }^{1-3}$

After Haiyan, NCD surveillance data were also limited. The Philippine Department of Health (DOH) has several health information systems and registries. The Field Health Services Information System collects information on different public health programmes and provides national health statistics. ${ }^{4}$ The Integrated Chronic Noncommunicable Disease Registry System is a web-based system that captures data from hospitals on NCDs such as cancer, diabetes, chronic obstructive pulmonary disease, stroke and renal disease. ${ }^{5}$ Primary health care (PHC) facilities, such as village health centres, have no access to this system. The Philippine Disease Surveillance and Response System is the routine disease surveillance system of
$\mathrm{DOH}$, established in compliance with International Health Regulations (2005). It captures data on 23 notifiable diseases and syndromes. ${ }^{6}$ The Surveillance in Post Extreme Emergencies and Disasters (SPEED) system provides real-time health information (both cases and deaths) on 21 syndromes, three of which are noncommunicable: high blood pressure, diabetes and acute asthma. SPEED is activated post-disaster and collects health information on a daily basis from facilities in disaster-affected areas. ${ }^{7}$ After Haiyan, SPEED was the only surveillance system that provided data on NCD surveillance.

As there were also no specific guidelines or protocols for NCDs post-disaster, the World Health Organization (WHO) Representative office in the Philippines supported the implementation of the WHO Package of Essential Noncommunicable Disease Interventions at the Primary Health Care (PEN) in Haiyan-affected areas. This support included training health providers and providing essential technologies (e.g. blood pressure measuring devices, stethoscopes and devices to determine blood glucose and cholesterol levels) to sustain NCD service delivery after Haiyan. ${ }^{8}$

In this brief report we provide the number of consultations and deaths from NCDs as reported from SPEED post-Haiyan and describe the effects that Haiyan had on people with NCDs as reported by front-line responders.

\section{METHODS}

We obtained the SPEED data set to identify the number of consultations and deaths for the noncommunicable

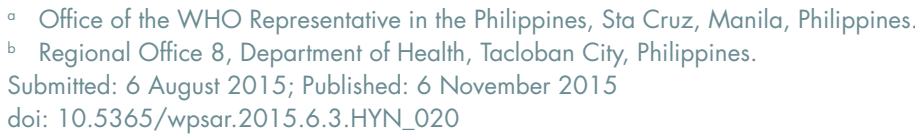




\section{Table 1. Outcomes of focus group discussions on NCDs by issue, Region 8, the Philippines, 2014}

\begin{tabular}{ccc}
\hline $\begin{array}{c}\text { What were the specific NCD } \\
\text { issues encountered? }\end{array}$ & $\begin{array}{c}\text { What are some of the things being done } \\
\text { to impact NCDs? }\end{array}$ & $\begin{array}{c}\text { What are your recommendations for future } \\
\text { disasters? }\end{array}$ \\
\hline
\end{tabular}

\section{Providers}

Totally or partially damaged facilities leading to interruption of health services

Building of temporary health facilities, some health facilities operate from tents and make-shift facilities
Lack of human resources in mostaffected areas mostly in Eastern Samar, some parts of Western Samar and Leyte

\section{Patients/consumers}

Stress (no medicines and drugs for NCDs, lack of food and clothing)

Life-saving medical services not available

\section{Logistics (supplies and equipment)}

Medical supplies at primary health care facilities and commercial pharmacies
$\mathrm{DOH}, \mathrm{PHO}$ and others deployed medical teams

Some nearby LGUs responded a day after the typhoon.

Provision of needs depending on the available resources

Respondent: "We're from the island, we transported patients to the mainland, we asked the Philippines Air Force to airlift the patients."

Medicines and medical supplies are solicited from other health facilities from other municipalities or provinces.
Respondent: "Emergency transport and communication facility should always be available in the province/region/nation." Another participant said that this should be integrated in the Health Emergency Preparedness, Response and Recovery Plans.

Respondent: "LGUs need to build a typhoon-resilient facility in case this will happen again in the future."

Respondent: "There is a need to create stand-by response teams, not only a response team, but one on basic life support and that has reliable equipment."

Respondent: "We need to stockpile ready-to-eat food packs for NCD patients." Another participants said we can use colour-coded food packs according to clients' conditions.

Respondent: "Establish helplines for air transport."

Respondent: "There is a need to develop emergency kits with laminated algorithm."

Respondent: There is a need for stockpiling of stocks, e.g. pre-pack one-month supply of medicines prior to the event (basic/first kit/ emergency NCD kit)."

Respondent: "There is a need for a MOA with local/ neighbouring pharmacy to make their medicines available to the LGU in case of emergency."

Respondent: "There is a need to develop field manuals that include NCDs so that when disaster comes we have guidelines."

Respondent: "SPEED is useful but HEMS add other NCDS on SPEED."

Respondent: "I wish there was a registry so that when a disaster comes we already know whom to help." morbidity and occurrences, e.g. Basey MHO said three died due to NCD, but the death was not

Establishing operation centre and adopting Health Emergency Management System

documented

DOH, Department of Health; HEMS, health emergency management staff; LGU, local government unit; MHO, municipal health office; MOA, memorandum of agreement; NCD, noncommunicable disease; PHO, provincial health office; and SPEED, surveillance on post extreme emergencies and disasters.

syndromes in Region 8 - high blood pressure, acute asthma attack and diabetes.

PEN implementation included training to determine the effects of Haiyan on people with NCDs. ${ }^{8}$ A focus group discussion was conducted with participants from Region 8 , the most-affected region. These participants, mostly front-line responders such as medical doctors, nurses, midwives and community health volunteers were grouped according to provinces; they were asked to discuss the effect Haiyan had on people with NCDs, what was being done and their recommendations for future disaster responses (Table 1 ). Thirty minutes were allocated with a facilitator moderating the session and group leaders assisting with each province group. Each group was asked to present their findings; content analysis was conducted to summarize participants' responses. 
Figure 1. Number of consultations reported on SPEED for noncommunicable syndromes by month, Region 8 , the Philippines, 8 November 2013 to 28 March 2014

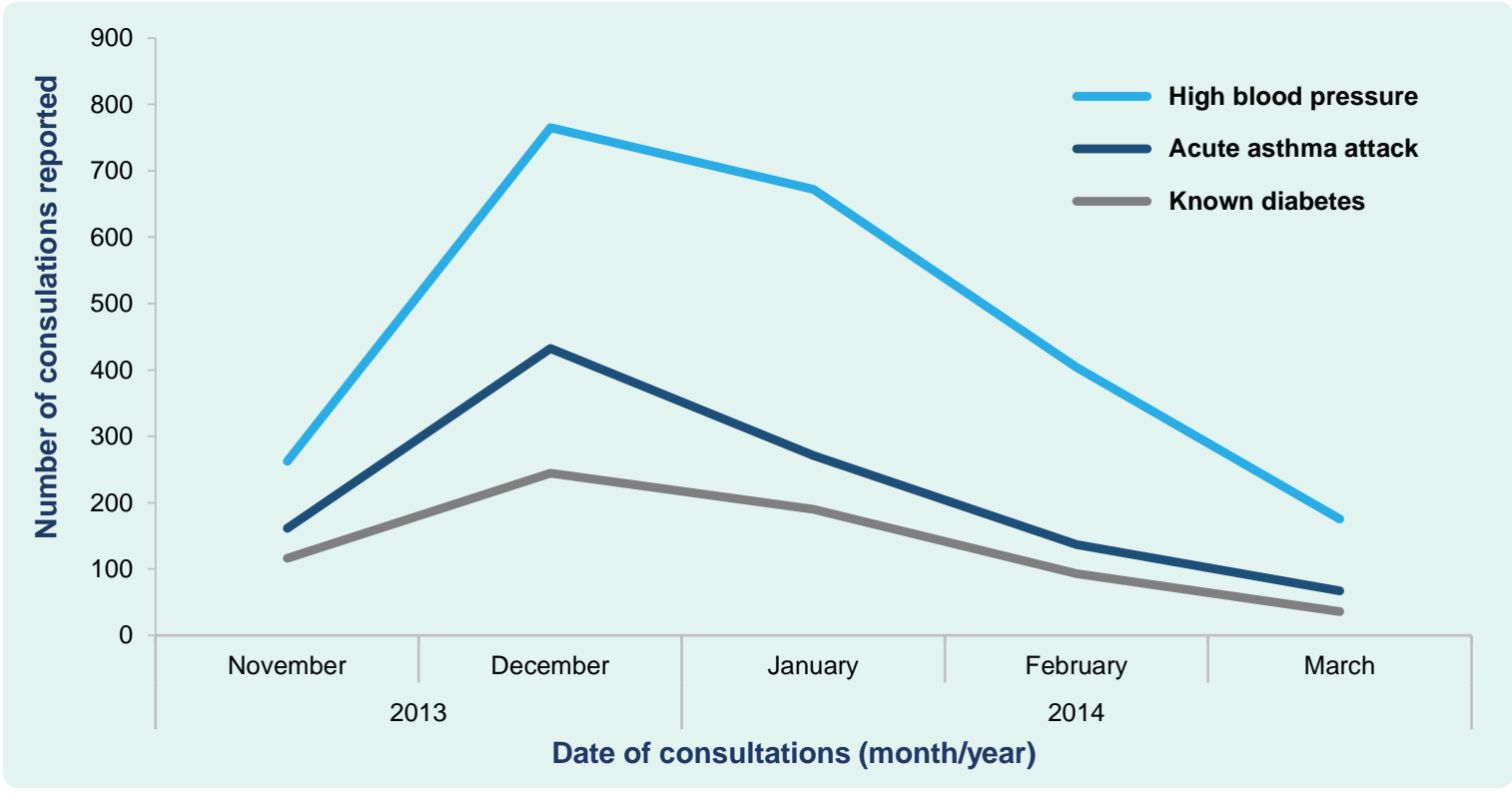

Source: Health Emergenc Management Staff (2014).

SPEED, Surveillance in Post Extreme Emergencies and Disasters.

\section{RESULTS}

SPEED reporting was conducted in 254 health facilities in four provinces of Region 8 (Leyte, West Samar, East Samar and Biliran) between 8 November 2013 and 28 March 2014. Most reports were from non-hospital facilities compared with hospitals (205/254, 80.7\% vs $49 / 254,19.3 \%$, respectively). High blood pressure (2278 cases), acute asthma attack (1068 cases) and diabetes (679 cases) were in the top 10 leading causes of SPEED consultations. This compared with 3194 consultations for acute respiratory infection, the most common reason for a consultation. No deaths from the three noncommunicable syndromes were reported in the system. The highest number of consultations for the three conditions were reported in December 2013 with decreasing numbers until March 2014 (Figure 1).

All provinces, cities and municipalities in Region 8 were represented in the focus group discussions. Of the nine conducted, there were three in Biliran Province; three in Leyte Island (in Leyte and South Leyte provinces); and three in Samar Island (one each in Samar, East Samar and North Samar provinces) with 10-15 participants per group. There were eight specific NCD issues including: damage to health facilities that interrupted usual services and lack of human resources; patient issues: stress with regards to inadequate medicines and other essentials and unavailability of lifesaving services; logistics issues: no stock of medicines and medical supplies; policy issues: lack of guidelines for NCDs; and surveillance issues: limited information and undocumented cases and deaths (Table 1).

Some responses to these issues included building temporary clinics, deploying health personnel and soliciting drugs and supplies from nearby provinces and municipalities. Recommendations for future responses included stockpiling food, drugs and medical supplies, developing a field handbook, and reviewing and revising of the Health Emergency Management Bureau surveillance package.

\section{DISCUSSION}

Our study suggested that high blood pressure, acute asthma attacks and diabetes were priority public health concerns following Haiyan. The number of consultations from SPEED for hypertension, asthma and diabetes were highest in December 2013, one month post-Haiyan, which could be due to the disrupted health-care system. The decrease in consultations after December 2013 
could be linked to the influx of foreign medical teams and local support in Region 8.

Although there were no deaths from the syndromes captured in SPEED, there were unofficial deaths from NCDs reported by focus group respondents. This could be because the deaths were not caused by the three syndromes included in SPEED or that NCD surveillance was not a primary concern during that time.

Our report has some limitations. As SPEED was the only surveillance system available post-Haiyan for NCDs, our analysis was limited to the three syndromes it includes. Also, the report focused only on Region 8 , which was the most-affected region. Therefore the data may not be representative of those in other regions.

In the Philippines, estimating the number of people with NCDs is challenging, especially post-disaster. Although SPEED provided some indication of the NCD burden post-Haiyan, we recommend also using an alternate surveillance system for collecting data on people with NCDs such as enhancing the Integrated Chronic Noncommunicable Disease Registry System. ${ }^{5}$ Since primary health care facilities had the most interaction with clients after Haiyan, they should be included in the system. Maintaining a NCD registry at the primary health care level is ideal and vital especially when related to the actual burden of disease and programme management.

Recommended strategies to improve NCD response in future disasters included strengthening primary health care and NCD management, including the development of guidelines and field manuals for health providers managing chronic diseases post-emergency. This included increasing research on NCDs morbidity and mortality patterns during and following emergencies and incorporating NCD-related protocols into existing emergency-related policies. Additional recommendations were to develop technical guidelines on the clinical management of NCDs in emergencies and integrating NCD-specific information into training for emergency workers and emergency-response coordinators. ${ }^{9}$

\section{Conflicts of interest}

None declared.

\section{Funding}

WHO Office in the Philippines.

\section{Acknowledgements}

This paper is dedicated to people in the Haiyanaffected areas, especially Eastern Visayas (Region 8) and international and local organizations that extended their help to Haiyan-affected areas. We thank our partners at the Department of Health Regional Office 8NCD unit with special mention to Dr Ma Sol Villones and Ms Mae Analyne Marquez. Special thanks to the WHO Representative office in the Philippines Team with special mention to $\mathrm{Dr}$ Julie Lyn Hall and to the WHO editing committee: $\operatorname{Dr}$ Allison Gocotano and Dr Megan Counahan.

\section{References}

1. Multi-cluster/sectoral initial rapid assessment (MIRA): Provisional version March 2012. Geneva, Inter-Agency Standing Committee., 2012 (http://interagencystandingcommittee.org/ system/files/legacy_files/2012_04_mira_manual.pdf, accessed 7 September 2015).

2. Multi-cluster initial rapid assessment (MIRA) - Nepal for multi-hazard scenarios as of July 2012. Katmandu, United Nations-Nepal, 2012 (http://un.org.np/sites/default/ files/2012-07-13-questionnaries-july-2012_0.pdf, accessed 7 September 2015).

3. Impact of natural disasters on the health system in Africa. Geneva, World Health Organization, 2009 (http://www.preventionweb. net/files/11214_WHOpresentationontheimpactofnatural.pdf, accessed 7 September 2015).

4. Field Health Services Information System: web update. Manila, Epidemiology Bureau, Department of Health, November 2014 (http://nec.doh.gov.ph/index.php?option=com_content\&view=arti cle\&id=59\&ltemid=78, accessed 7 September 2015).

5. Integrated chronic noncommuncable disease registry system (ICNCDRS) - manual of operations. Manila, Department of Health, 2012 (http://chronic.doh.gov.ph/forms/ICNCDRS\%20Manual\%20 of\%200perations.pdf, accessed 7 September 2015).

6. Gallardo FDL et al. An assessment of the case notification system 16 months after Typhoon Haiyan, Region 8, the Philippines. Western Pacific Surveillance and Response Journal, 2015, 6(Suppl 1):71-75. doi:105365/wpsar.2015.6.2.HYN_002

7. Surveillance in Post Extreme Emergencies and Disasters: operations manual for managers. Manila, Department of Health, 2011 (http://www.wpro.who.int/philippines/publications/speed operations_manual.pdf, accessed 7 September 2015).

8. Martinez RE et al. Use of WHO Package of Essential Noncommunicable Disease Interventions after Typhoon Haiyan. Western Pacific Surveillance and Response Journal, 2015, 6 (Suppl 1):18-20. doi:10.5365/wpsar.2015.6.3.HYN_024

9. Demaio A et al. Noncommunicable Diseases in Emergencies: Call to Action. PLOS: Currents Disasters, 2013, Edition 1. doi:10.1371/currents.dis.53e08b951d59ff913ab8b9bb51 c4d0de 